UDC 621.396.6

\title{
A NOVEL APPROACH TO WEAK SIGNAL PROCESSING
}

\author{
Oleksandr S. Makarenko, Oleksiy V. Melnichuk \\ National Technical University of Ukraine “KPI”, Kyiv, Ukraine
}

A novel effective approach to weak continuous and digital signals processing in communication channels with high noise levels is proposed and investigated. The essence of proposed approach is illustrated by examples of various electronic devices. The conditions which allow obtaining the maximal gain in signal to noise ratio are found. This gain exceeds $50 \mathrm{~dB}$ for sensitive amplifier and approximately equals to $30 \mathrm{~dB}$ for detector of digital cosine video impulse. It is shown that the proposed approach ensures obtaining a lot more gain in signal to noise ratio compared to existing technical solutions. The considered advantages of the described devices allow employing the proposed approach to effective information processing in communication systems with low operating power.

\section{Introduction}

The modern technological advances are largely determined by extensive use of various communication systems. Usually, the electromagnetic compatibility requirements dictate the transmitter power limitation. In the case of lower transmitted power, the necessary signal level is achieved by increasing the receiver sensitivity. Typically, the receivers with high sensitivity are composed of significant number of amplification stages. Therefore, the power of desired signals is commensurable with the level of intrinsic noises. This creates considerable difficulties in processing of these signals [1]. There is the problem of detecting the weak signals from the additive mixtures of signals and noises also for digital communication systems [2,3]. For detection of cosine video impulses in digital communication systems, the optimal filters or low-pass filters with optimal bandwidth (quasi-optimal filters) are applied [3].

For weak signals processing in strong noises, a large number of methods were developed. The methods of optimal filtering using the various criteria of optimality are widely applied [4]. The criteria of maximal signal to noise ratio, minimum of average error square and maximum of a posteriori probability are most commonly used. Optimal filters are well understood and described in the literature [5]. It should be noted that the power gain in signal to noise ratio, when using the optimal filtering for signal detection, is equal to the signal base $M=T_{s} \cdot \Delta f$, where $T_{s}$ denotes the signal duration and $\Delta f$ is the frequency band in which it is transmitted. When using the quasi-optimal filtering in analog devises, this gain is less on $0.9 \mathrm{~dB}$ [5].

The purpose of this paper is the development of novel technique of weak signal processing in strong noise condition and its application to the creation of sensitive amplifier and detector of cosine impulses to be applied in digital communication systems. To solve the named problem, the paper discusses and substantiates the essence of proposed technique of desired signal detection on strong noise background, describes the operation of sensitive amplifier and detector of cosine video impulses, reveals advantages and areas of the described devices application.

\section{A technique of weak signal processing in strong noise condition}

To overcome the limitations of existing methods based on optimal filtering, we propose a novel technique of weak signal processing in strong noise condition allowing us to reach much higher values of signal to noise ratio. The essence of the technique proposed consists in the following. The desired signal $f(t)$ with the noise is amplified. This amplified mixture exercises the amplitude modulation of the carrier frequency oscillation. The received oscillation is limited by means of the fast-response nonlinear circuit. At that, the positive half-period of amplitude-modulated signal and positive peaks of noise are extracted on resistive load.

The obtained total oscillation, containing the sequence of cosine impulses and noise, is passed through a filter. The filter cutoff frequency is chosen from the condition of undistorted transmission of cosine impulses. After such filtering, the mixture of signal and noise undergoes a doubled differentiation. As a result, the investigated signal is converted into the sequence of cosine impulses of opposite sign. Each of these cosine impulses is in equal measure weakened and contains two delta functions, which are eliminated. At the same time, the noise becomes the high-frequency one because under noise doubled differentiation its power spectral density is changed proportional to $\omega^{4}$, since 
the power spectral density of the noise on the output of linear four-pole circuit is equal to its input power spectral density multiplied by square of this circuit transmission coefficient modulus [4]. The obtained mixture is supplied to Butterworth low-pass filter of fourth order. This filter allocates the signal $f(t)$ and significantly attenuates the noise.

\section{Analysis of sensitive amplifier}

The described technique of weak signal processing in strong noise condition has been applied to the development of sensitive amplifier. The block diagram of considered sensitive amplifier is presented in Fig. 1.

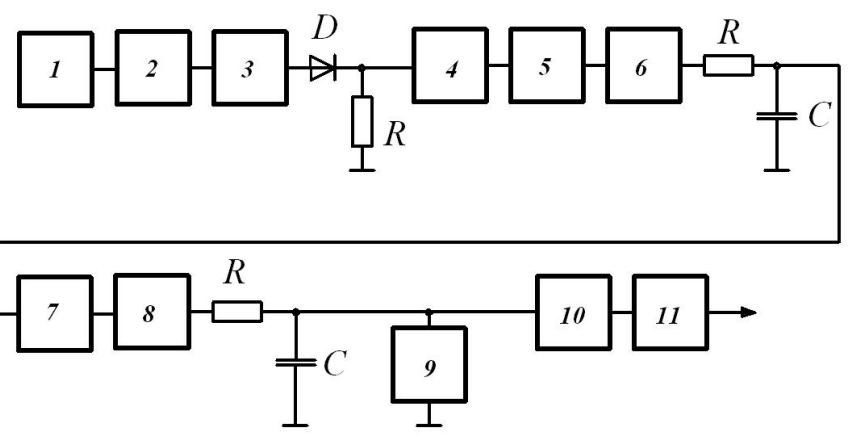

Fig. 1. Block diagram of sensitive amplifier: (1) source of the signal with the noise; (2) amplifier; (3) modulator; (4) filter; (5) differentiator; (6) isolation amplifier; (7) differentiator; (8) isolation amplifier; (9) electronic key; (10) low-pass filter; (11) amplifier-inverter.

Let us consider the operation principle of sensitive amplifier. The signal of source $l$ with internal resistance $R_{s}$ is amplified to the desired level by amplifier 2. Both the amplified signal and the oscillation of carrier frequency $f_{0}$ are supplied to the amplitude modulator 3. From the modulator output, the signal is supplied to the inertialess nonlinear circuit in the form of diode $D$ with resistive load $R$. This circuit forms a sequence of cosine impulses which amplitude is varied according to the law of control signal $f(t)$. Thus, the input of the Butterworth filter of second order 4 receives the sequence of cosine samples satisfying the Kotelnikov theorem. Fig. 2 illustrates the named sequence of cosine samples.

The cutoff frequency of the filter 4 is selected from the condition of undistorted transmission of cosine impulse with duration $T_{s}=T_{0} / 2$, where $T_{0}=1 / f_{0}$. The form of cosine impulse shown in Fig. 3 is described by the expression $S(t)=A \cos \left(\pi t / T_{s}\right),-T_{s} / 2 \leq t \leq T_{s} / 2$, where $A$ is impulse amplitude and $T_{s}$ denotes its duration. The spectrum of such impulse can be written in the form [3]

$$
S(f)=2 A T_{s} / \pi \cdot \cos \left(\pi f T_{s}\right) /\left[1-4\left(f T_{s}\right)^{2}\right] .
$$

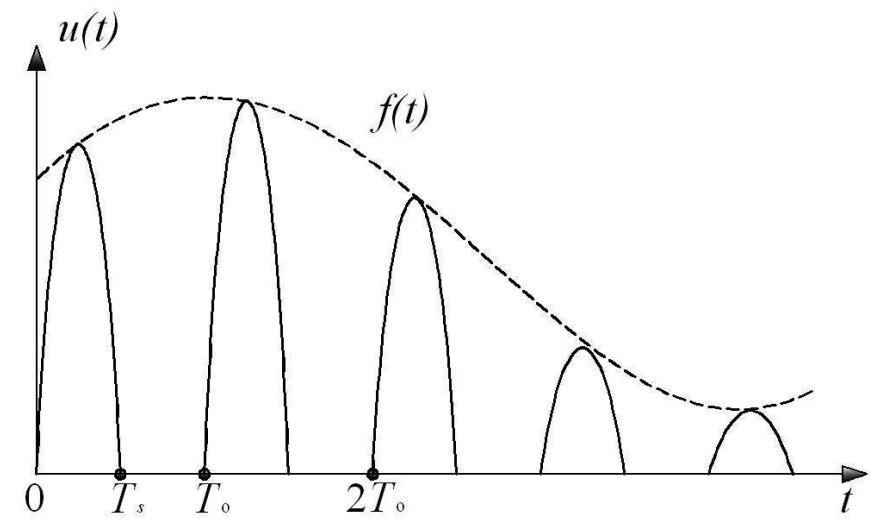

Fig. 2. The sequence of cosine samples.

The chart of this impulse spectrum is shown in Fig. 4. With accuracy sufficient for practical purposes, one can assume that the impulse spectrum is concentrated in the frequency band $0-f_{\text {aver }}$, where $f_{\text {aver }}=3 /\left(2 T_{s}\right)$. Since $T_{s}=T_{0} / 2$ then $f_{\text {aver }}=3 f_{0}$.

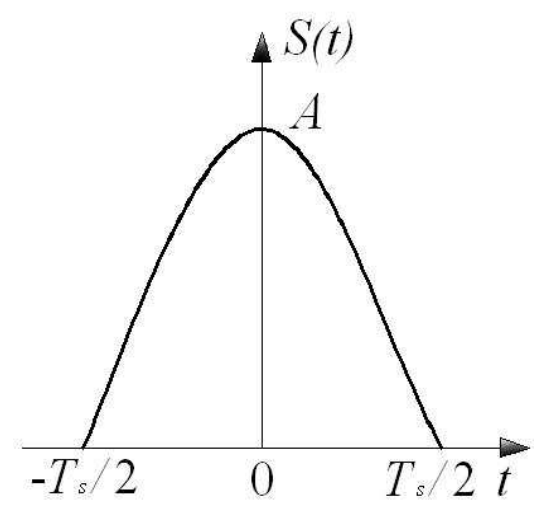

Fig. 3. Cosine impulse.

As a filter 4 , it is advisable to use the Butterworth filter of second order passing the signals in frequency band $0-f_{\text {aver }}$. The Butterworth filter of second order provides the decline slope of amplitude-frequency characteristic of $12 \mathrm{~dB}$ per octave that allows reducing the noise level outside the pass band to the necessary extent. Both the noise of input signal source and noise of amplifier are independent and centered therefore they are summarized in power on the output of the filter 4 [6].

Further, the mixture of signal and noise undergoes a doubled differentiation. Since the circuit from input of the filter 4 to output of considered sensitive amplifier is linearly parametric, the superposition principle can be applied. According to this principle, passing the signal and the noise through processing device can be calculated separately. 


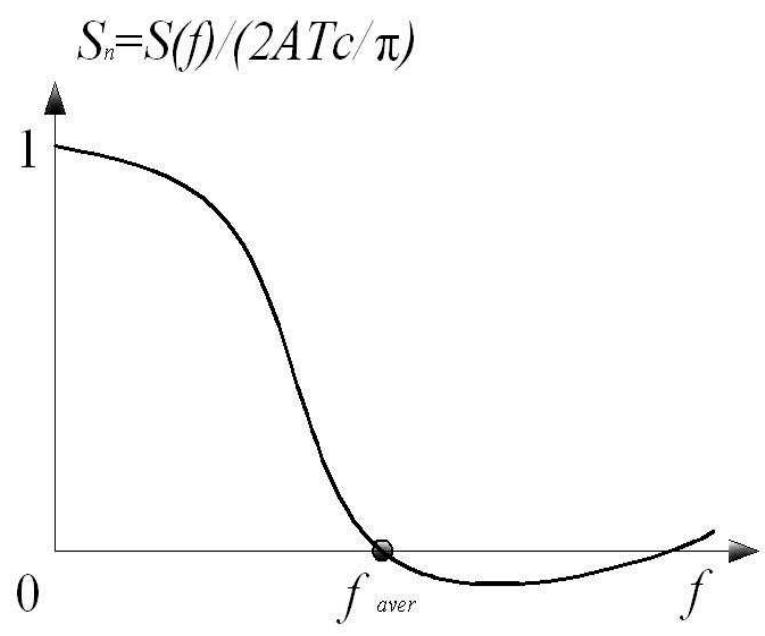

Fig. 4. Cosine impulse spectrum.

From output of the filter 4 through the first differentiator 5, signal or noise enter input of the first isolation amplifier 6 and further they enter input of restriction low-pass filter with cutoff frequency $3 f_{0}$. From the output of this $R C$ filter, signal or noise are successively passed through the second differentiator 7 , the second isolation amplifier 8 on input of the second restriction $R C$ filter with cutoff frequency $f_{\text {aver }}=3 f_{0}$. The capacity $C$ of this filter can be short-circuited by means of electronic key 9. As it is shown below, this allows eliminating delta functions generated during the doubled differentiation of the signal. From the output of second restriction $R C$ circuit through low-pass Butterworth filter 10 of fourth order, the signal enter the input of amplifier-inverter 11 finishing the processing device.

The $R C$ circuits are included into processing device in order to restrict the noise increase on outputs of isolation amplifiers because of their differentiation. A sequence of control impulses is supplied on the electronic key 9 making possible to eliminate delta functions of signal. These impulses are generated by control circuit from carrier frequency oscillations creating by separate generator of frequency $f_{0}$.

\section{Theoretical justification of processing technique}

Let us consider the noise suppression in processing device. It is known [7] that spectral density of noise power after doubled differentiation is expressed by $G_{\text {out }}=G_{0}\left(K_{r} \tau_{d} \omega\right)^{4}$, where $G_{0}$ denotes the uniform energetic spectrum of the noise on output of the filter; $K_{r}$ designates the resulting transmission coefficient of the differentiator and isolation filter; $\tau_{d}$ is the time constant of the differentiator chosen from the condition $\tau_{d} \omega_{\text {aver }}=0.1$.
The noise dispersion at output of the low-pass filter 10 can be expressed as follows [7]:

$$
\begin{aligned}
P_{\text {out }}=\sigma_{\text {out }}^{2} & =1 /(2 \pi) \int_{0}^{\omega_{\text {ower }}} G_{0}\left(K_{r} \tau_{d} \omega\right)^{4} K^{2}(\omega) d \omega \approx \\
& \approx 1 /(10 \pi) \Omega_{m} G_{0}\left(K_{r} \tau_{d} \Omega_{m}\right)^{4},
\end{aligned}
$$

where $G_{0}$ denotes the energetic spectrum on input; $\left(K_{r} \tau_{d} \omega\right)^{4}$ designates the square of transmission coefficient modulus of two differentiators with isolation amplifiers; $\Omega_{m}$ is the cutoff frequency of low-pass Butterworth filter of fourth order which is equal to maximal frequency in the input signal spectrum; $K^{2}(\omega)$ defines the square of transmission coefficient modulus of lowpass Butterworth filter of fourth order wherein it is equal to $K^{2}(\omega) \approx 1$ in the frequency band from 0 to $\Omega_{m}$ and it is equal to zero beyond boundaries of this band.

Without doubled differentiation, the noise power on output of the low-pass filter can be present as

$$
\sigma^{2}=1 /(2 \pi) \int_{0}^{\omega_{\text {ower }}} G_{0} K^{2}(\omega) d \omega \approx 1 /(2 \pi) G_{0} \Omega_{m} .
$$

Then, the power odd coefficient for the noise can be obtained in the form

$$
K_{n}=\sigma^{2} / \sigma_{\text {out }}^{2}=5 /\left(K_{r} \tau_{d} \Omega_{m}\right)^{4} .
$$

Let us introduce the condition that the noise power on input of the low-pass filter 10 at doubled differentiation would be the same as on output of filter 4 . Since $\tau_{d} \omega_{\text {aver }}=0.1$ and $5 /\left(K_{r} \tau_{d} \omega_{\text {aver }}\right)^{4}=1$, we find that $K_{r}=10(5)^{1 / 4}$. Then, the expression (3) is reduced to the form:

$$
K_{n}=\left(\omega_{\text {aver }} / \Omega_{m}\right)^{4} .
$$

Let us consider passing the signal through processing device. After first differentiation, the signal has the form shown in Fig. 5 and can be described by relation

$$
S^{\prime}(t)=-A\left(\pi K_{r} \tau_{d} / T_{s}\right) \sin \left(\pi t / T_{s}\right) .
$$

The second differentiation transforms the signal in accordance with following expression:

$$
\begin{gathered}
S^{\prime \prime}(t)=-A\left(\pi K_{r} \tau_{d} / T_{s}\right)^{2} \cos \left(\pi t / T_{s}\right)+A\left(\pi K_{r} \tau_{d} / T_{s}\right) \times \\
\times\left[\delta\left(t+T_{s} / 2\right)+\left(t-T_{s} / 2\right)\right] .
\end{gathered}
$$




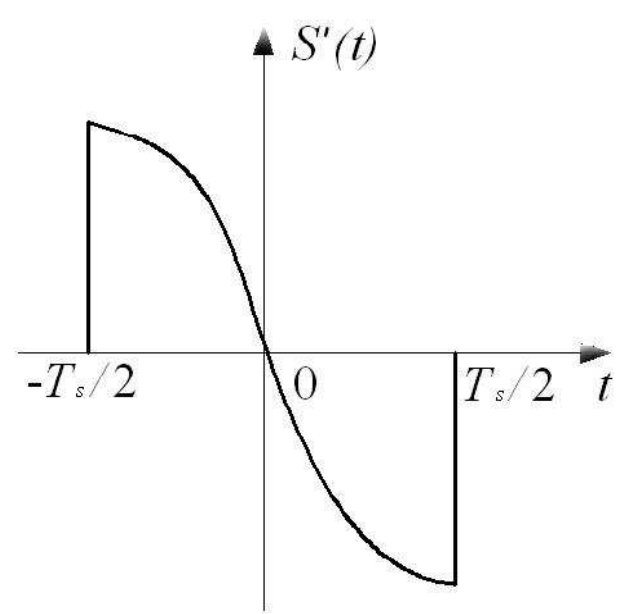

Fig. 5. Signal after first differentiation.

The signal form is illustrated by Fig. 6. According to this figure and using relations (5)-(6), we can find the degree of signal attenuation in device taking into account the delta function elimination by means of electronic key 9 shorting the capacity of second restricting filter for a short time. As a result, we get the following value of signal attenuation coefficient

$$
K_{\text {att }}=\left(\pi K_{r} \tau_{d} / T_{s}\right)^{2}=5^{1 / 2} / 9 .
$$

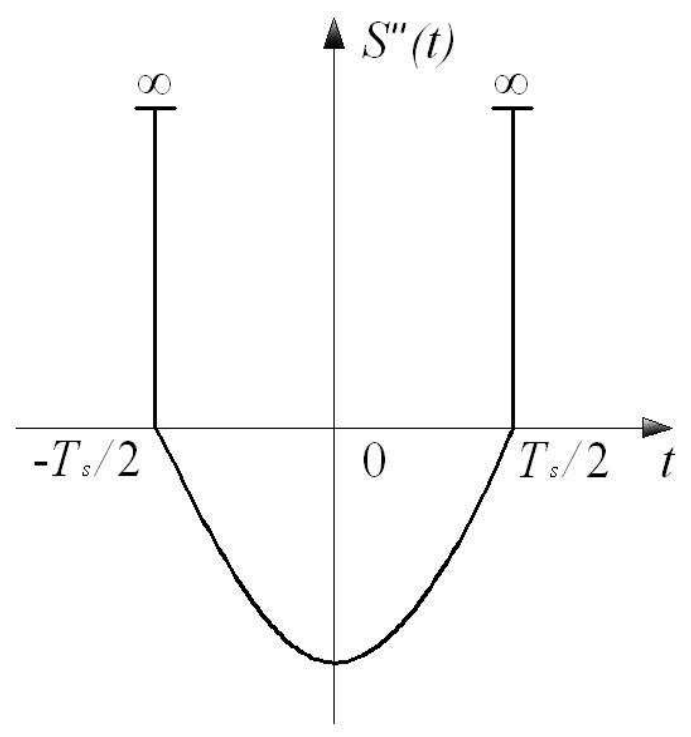

Fig. 6. Signal form after second differentiation.

Thus, the power signal attenuation is $12 \mathrm{~dB}$ under its doubled differentiation. One can show that the signal samples attenuation constitutes $10 \mathrm{~dB}$ in output filter 10. Thus, the summarized attenuation of the signal in processing device is $22 \mathrm{~dB}$.

The noise attenuation is defined by expression (4) and depends from relation $\omega_{\text {aver }} / \Omega_{m}$. It is obvious that $K_{n}$ can be significant with an appropriate choice $\omega_{0}=\omega_{\text {aver }} / 3$. For instance, when $F_{m}=\Omega_{m} /(2 \pi)=10$ $\mathrm{kHz}, f_{0}=300 \mathrm{kHz}, f_{\text {aver }}=900 \mathrm{kHz}$, the noise attenuation coefficient $K_{n}=78 \mathrm{~dB}$. Taking into account the signal attenuation, the gain with respect to the signal to noise ratio is $K_{g}=78-22=56 \mathrm{~dB}$. The attenuated signal is amplified and inverted by means of low noise amplifier-inverter 11 installed at output of the low-pass filter 10 .

It should be noted that cosine impulse is only one of known signals which retains own shape up to sign under doubled differentiation. It contains two delta functions of opposite sign which can be eliminated. In contrast to signal, the noise under doubled differentiation has energetic spectrum changing proportionally to $\omega^{4}$ becoming high frequency that provides background for its significant attenuation by means of output low-pass filter 10 as shown in Fig. 7.

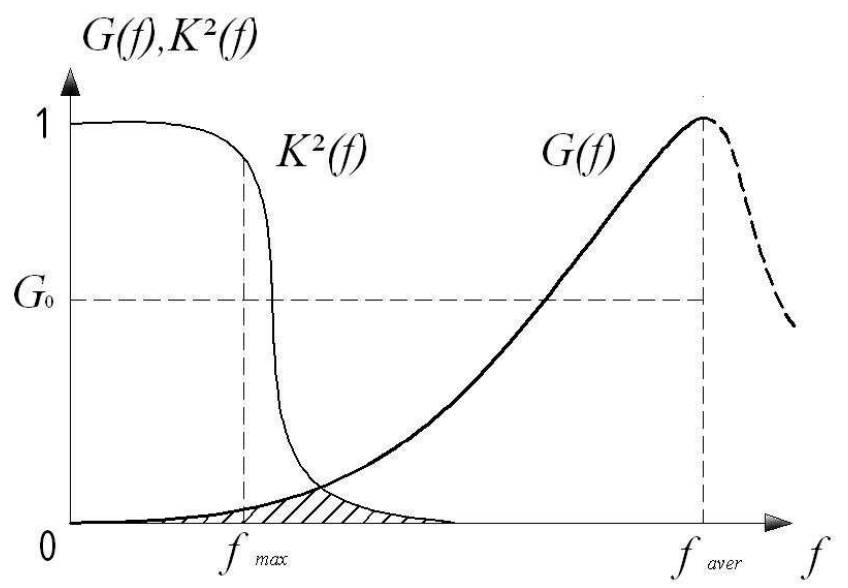

Fig. 7. Illustration of noise attenuation by means of low-pass filter: $K^{2}(f)$ denotes the square of transmission coefficient modulus of Butterworth filter of fourth order; $G_{0}$ designates the spectral density of noise power before differentiation; $G(f)$ defines the spectral density of noise power after doubled differentiation; hatched region under curves denotes the noise power at output of low-pass filter 10 indicating a significant reduction of noise power at output of processing device.

Fig. 8 presents the impulse sequence for controlling the operation of electronic key 9 eliminated the delta functions.

\section{Detector of cosine video impulses}

Let us consider the detector of weak cosine video impulses widely used in digital communication systems [3]. The block diagram of considered detector is presented in Fig. 9. In contrast to sensitive amplifier which block diagram is shown in Fig. 1, the detected video impulse enters the input of filter 2 from output of receiver demodulator 1 . Moreover, the pass band of low- 
pass filter 7 is chosen from conditions of obtaining the maximal signal to noise ratio and making the minimal inter-symbol distortions [6].

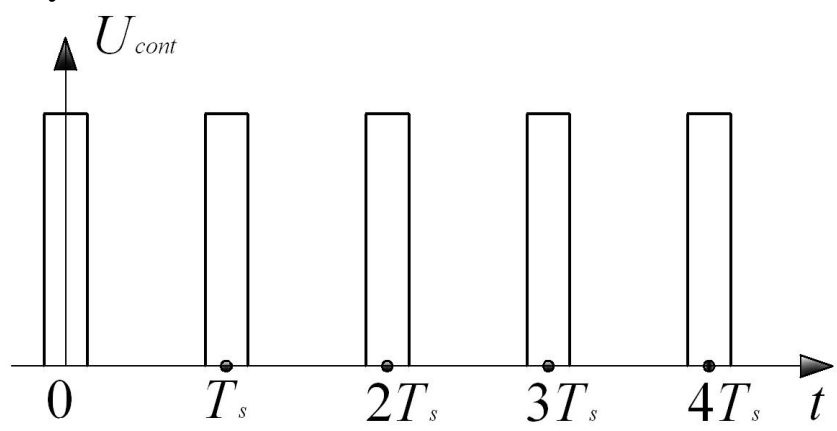

Fig. 8. Sequence of control impulses.

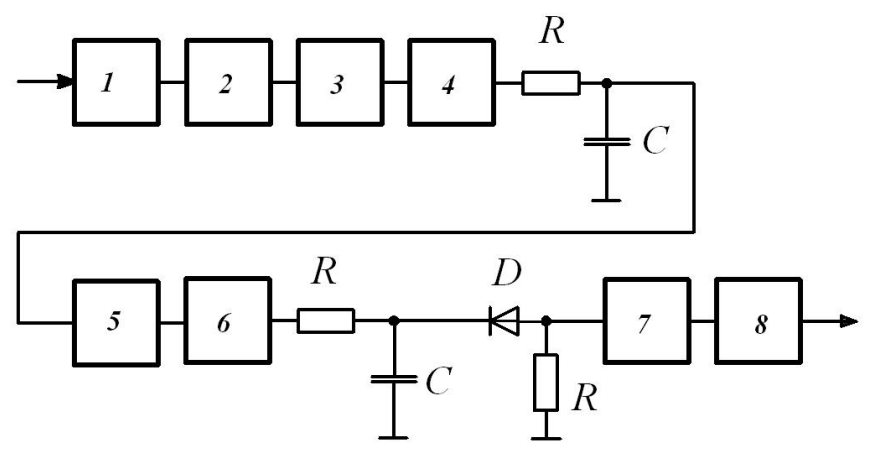

Fig. 9. Block diagram of cosine impulses detector: (1) synchronous demodulator; (2) filter; (3) differentiator; (4) isolation amplifier; (5) differentiator; (6) isolation amplifier; (7) low-pass filter; (8) amplifier-inverter.

To eliminate the delta functions generated by doubled differentiation, the inertia-less nonlinear circuit consisting of diode $D$ with resistive load $R$ is installed between output of second isolating filter and input of low-pass filter 7 instead of electronic key requiring the synchronous control. Because the positive noise releases are truncated by diode and do not passed further, the additional gain in signal to noise ratio equaled to $3 \mathrm{~dB}$ is guaranteed.

The results of MATLAB computation show that the optimal pass band of output low-pass filter 7 is $20 \mathrm{kHz}$ for cosine video impulses at following values of parameters: $T_{s}=10 \mu \mathrm{s}, f_{\text {aver }}=150 \mathrm{kHz}$. The resulting gain in signal to noise ratio is $15 \mathrm{~dB}$ that is substantially better than in existing detection systems with optimal filtering or quasi-optimal filtering at the signal basis $M=1.5$. The gain in signal to noise ratio at optimal filtering with $M=1.5$ constitutes $10 \log M=1.76 \mathrm{~dB}$.

It is also possible to use the fourfold differentiation of signal and noise mixture. Of course, this leads to a complication of processing device, however, it allows obtaining the gain in signal to noise ratio exceeding 30 $\mathrm{dB}$. Application of such detectors in modern digital communication systems will significantly reduce the powers of transmitters of cell phones and base stations of mobile communication systems.

\section{Conclusion}

A novel effective approach to weak continuous and digital signals processing in communication channels with high noise levels is proposed and investigated. The essence of proposed approach is illustrated by examples of sensitive amplifier and detector of digital cosine video impulses. It is shown that the proposed approach ensures obtaining a lot more gain in signal to noise ratio compared to existing technical solutions. In the electronic equipment based on proposed technique, there is a significant noise attenuation not only of amplifiers prior to processing units but also noise of input signal source. It is a prerequisite for the creation of electronic equipment with new functional possibilities. The proposed technique can be successfully applied in digital communication systems which employ the quadrature processing circuit with synchronous detectors. The considered advantages of the described devices allow employing the proposed approach to effective information processing in communication systems with low operating power including cell phones and base stations of mobile communication systems.

It is possible to implement the proposed technique for creating the modern electronic equipment of satellite communication systems, radiolocation, radio navigation, and various diagnostic systems.

\section{References}

1. Noghin V. N. Analog electronic devises. - Moscow: Radio and Svyaz, 1992. - 304 p. [in Russian].

2. Sklar B. Digital communications: Fundamentals and applications. - Hardcover: Prentice Hall, 2001. - 1080 p.

3. Galkin V. A. Digital mobile radio communications. Moscow: Hotline - Telecom, 2007. - 432 p. [in Russian].

4. Gonorovsky I. S. Radio circuits and signals. - Moscow: Soviet Radio, 1977. - 608 p. [in Russian].

5. Lezin Yu. S. Introduction to theory and technique of radio systems. - Moscow: Radio and Svyaz, 1986. - 280 p. [in Russian].

6. Lathi B. P. Information transmission systems. - Moscow: Svyaz, 1971. - 324 p. [in Russian].

7. Tikhonov V. I. Statistical radio techniques. - Moscow: Radio and Svyaz, 1982. - 624 p. [in Russian].

8. Makarenko O. S. Detection device of cosine digital and radar signals // Patent of Ukraine No. 75231 from November 26, 2012 [in Ukrainian].

Received in final form January 19, 2013 